\title{
An efficient approach for computing analytical non-parametric fragility curves
}

\author{
Domenico Altieri ${ }^{1}$, Edoardo Patelli ${ }^{1 *}$ \\ ${ }^{1}$ Institute for Risk and Uncertainty, University of Liverpool, Peach Street, Chadwick \\ Building, Liverpool L69 7ZF,UK, \\ D.Altieri@liverpool.ac.uk,Edoardo.Patelli@liverpool.ac.uk
}

\begin{abstract}
Fragility curves are used in earthquake engineering for assessing the seismic vulnerability of structures or systems. Direct estimations of fragility curves by means of simulation-based approaches lead generally to relevant computational costs, especially when the failure region is characterized by small probabilities of occurrence. Simplified hypotheses are therefore introduced in the common practice to approximate the dependency between the structural response and the associated seismic intensity level.

The study proposes a non-parametric methodology to estimate analytical fragility curves without specific assumptions on their final shape. The approach starts by identifying all the subsets characterized by the same values of the chosen seismic intensity measure parameter. Then, the failure region is mapped by means of a classification algorithm coupled with a polynomial kernel. Finally, the conditional failure probability is computed by associating the samples generated in each subset to the corresponding classification score. A stochastic earthquake model is employed to define the seismic dataset and the uncertainty associated with the ground motion records.

Two case studies are analyzed in which the non-parametric methodology is compared against three popular parametric approaches and a reference solution. The proposed approach shows an overall higher accuracy and efficiency, especially in case of rare failure domains.
\end{abstract}

Keywords: Seismic fragility curves, Conditional failure probability, Reliability analysis, Performance based earthquake engineering 


\section{Introduction}

Seismic fragility curves describe how the failure probability of structural systems or components evolves for different given levels of a seismic intensity measure parameter (IM) adopted to characterize the ground motion record (e.g. peak ground acceleration, spectral acceleration at the fundamental frequency). They are gaining popularity especially in the last years due to their importance in the framework of a performance based earthquake engineering (PBEE) approach $[1,2]$. For example, by combining the fragility curve and the seismic hazard curve it is possible to compute the mean annual rate of the failure state $[3,4]$.

The dataset of the structural response under different seismic actions, needed to compute fragility curves, can be obtained from both empirical data (observed failures) (e.g. $[5,6])$ and analytical model evaluations (e.g. $[7,8]$ ). In the first case data are collected by recording the damage state of a set of structures along with the intensity seismic levels, that can be estimated from a ground motion instrument or in absence of that from a ground motion map $[9,10]$ or a proper attenuation law.

In case of analytical fragilities curves (i.e. computed on the basis of nonlinear dynamic analyses), different procedures have been proposed to efficiently perform nonlinear dynamic structural analyses for increasing seismic intensity measures. Classic analytical parametric methodologies are based on the assumption that fragility curves can be defined by means of a lognormal cumulative distribution function so that two parameters, median $\hat{\mu}$ and standard deviation $\hat{\beta}$, are sufficient to derive conditional seismic reliability measures for any given failure criterion $[11,12,13]$. The statistical parameters $\hat{\mu}$ and $\hat{\beta}$ refer to the dataset of $I M s$ corresponding to the failure state, since the randomness of the seismic input can lead to different dynamic response even having the same $I M$. The estimation of $\hat{\mu}$ and $\hat{\beta}$ is commonly obtained by means of three different approaches: method of moments, maximum likelihood method or by adopting a regression demand model (mostly linear) in the log-scale between the $I M s$ and the structural response $[14,15]$. One of the most common parametric approach performs a sequence of dynamic analysis by repeatedly scaling the ground motion until the failure state is reached (Incremental Dynamic Analysis - IDA) [11]. However, IDA may require significant computational efforts and the final fragility curve may be affected by the scaling stage since the ground motion frequency content may be not representative of the corresponding intensity level $[16,17]$. In this 
regard, [18] proposes an hybrid procedure to reduce the number of analysis required by a classic IDA by previously estimating the seismic intensity level corresponding to the failure state by means of the so-called Cloud Analysis $[19,13]$ that provides a simple linear relation between the un-scaled seismic inputs and the corresponding Engineering Demand Parameter $(E D P)$ values. Alternatively, the so-called Multiple Stripes Analysis (MSA) allows estimating the median and the dispersion of a sequence of 'stripes' of structural responses for increasing constant $I M s$, until the collapse is reached along the whole stripe $[20,21]$.

In general, parametric fragility models provide a smooth fragility curve easy to estimate. However they can lead to a lack of fit with respect the real data distribution due to the restriction on the final shape [22]. While parametric methods have been widely addressed due their robustness and simplicity, this is not the case for non-parametric procedures that result mainly based on the use of kernel smoothing methods (KSMs) $[22,23,24]$ or the copula function [25]. These numerical approaches turn out to be extremely flexible and efficient to compute the joint probability $P(E D P, I M)$ or the conditional probability $P\left(E D P \mid I M=i m_{i}\right)$ for the fragility curve estimation. However, KSMs can suffer of over-fitting or show bias near the boundaries of the analyzed domain, while a copula function cannot be always properly fitted and the selection of the optimal copula remains somehow murky [26]. The approach introduced in this article does not work directly on the joint probability $P(E D P, I M)$ and does not require an ambiguous tuning stage. More specifically, this study proposes a methodology able to derive seismic fragility curves without any assumption on the final conditional cumulative density function, through a failure region mapping process that employs a Multinomial Logistic Regression coupled with a polynomial kernel. The absence of a predefined final shape reduces the risk of lack of fitting, while the use of probabilistic scores instead of a classical indicator function makes the approach more flexible and applicable even in case of noise along the failure region.

The paper is organized as follows. Section 2 provides a brief description of three parametric approaches employed to validate and test the proposed procedure. In section 3 the proposed methodology is described into details along with the failure region mapping process. In section 4 the problem raised by the seismic dataset selection is presented and two alternatives discussed. In section 5 two different case studies are presented and finally, all the results are reported and the conclusions drawn. 


\section{Fragility curves and parametric models}

Analytical fragility curves allow selecting the $I M$ levels for the seismic dataset definition along with the number of dynamic analysis required for each $I M$ value [12]. This aspect contributes to define methods increasingly able to perform an efficient dataset selection by reducing the number of analyses performed.

Generally, by assuming that the IMs causing the failure are lognormal distributed then the final fragility curve can be derived from just 2 parameters, the median capacity $\hat{\mu}$ (i.e. the $I M$ level associated to a probability of failure of 0.5$)$ and the dispersion measure $\hat{\beta}$. The fragility curve can therefore be expressed as $[11,12,13]$ :

$$
P\left(\text { Failure } \mid I M_{i}\right)=\Phi\left(\frac{\log I M_{i} / \hat{\mu}}{\hat{\beta}}\right)
$$

where $\Phi$ represents the standard normal cumulative density function.

A brief overview about three of the most established parametric procedures based on the lognormal assumption is provided in this section.

\subsection{Incremental Dynamic Analysis}

Incremental dynamic analysis computes the structural response for a sequence of scaled ground motion records until the failure state is reached [11]. Thus, for any given failure criterion, a dataset of seismic intensity measures corresponding to the failure can be obtained trough a linear interpolation and subsequently assumed as lognormal distributed. The fragility curve parameters $\hat{\mu}$ and $\hat{\beta}$ are obtained by fitting the interpolated $I M_{i}$ through the method of moments [27]:

$$
\log \hat{\mu}=\frac{1}{n} \sum_{i=1}^{n} \log I M_{i}
$$

$$
\hat{\beta}=\sqrt{\frac{\sum_{i=1}^{N}\left(\log I M_{i}-\log \hat{\mu}\right)^{2}}{N-1}}
$$

where $N$ is the number of ground motion records considered in the analysis. For the scaling of the initial seismic dataset, instead of simply using a constant incremental step until the failure occurs, a more efficient algorithm is employed in this study allowing to control at the same time the tolerance resulting from the final linear interpolation [11]. 


\subsection{Multiple Stripes Analysis}

Similarly to the $I D A$, the Multiple Stripe Analysis (MSA) [20, 21] performs a sequence of dynamic analyses for different seismic datasets, each one corresponding to a fixed $I M$ level. The final 'stripes' are therefore characterized by a constant $I M$ level vs multiple structural responses. The method considers increasing $I M$ levels until the failure is reached for the whole intermediate seismic dataset. The final fractions of analyses causing failure can be fitted using the maximum likelihood method. In particular, the logarithmic median $\hat{\mu}$ and standard deviation $\hat{\beta}$ are computed as following:

$$
\{\hat{\mu}, \hat{\beta}\}=\max _{\mu, \beta} \prod_{i=1}^{N_{I M_{i}}}\left(\begin{array}{c}
n_{i} \\
f_{i}
\end{array}\right) \Phi\left(\frac{\log I M_{i} / \mu}{\beta}\right)^{f_{i}}\left(1-\Phi\left(\frac{\log I M_{i} / \mu}{\beta}\right)\right)^{n_{i}-f_{i}}
$$

where, considering $N_{I M_{i}}$ number of intermediate $I M$ levels, $n_{i}$ and $f_{i}$ represent the corresponding number of collected ground motion records and system failures respectively.

To reduce the computational effort the procedure can be stopped before the whole dataset causes the failure, affecting the final accuracy, or alternatively the median demand for a given $I M$ and the associated dispersion can be computed from the percentiles of the stripes, as described in [13].

\subsection{Cloud Analysis}

The method employs a simple conditional $I M$-based approach by defining a linear probabilistic model in the logarithmic space after computing the EDP values for a set of ground motion records characterized by different $I M$ levels $[19,13,28]$. The probabilistic model is defined as following:

$$
\log \hat{\mu}_{Y \mid I M}=\log a+b \cdot \log I M
$$

$$
\hat{\beta}_{\log Y \mid I M}=\sqrt{\frac{\sum_{i=1}^{N}\left(\log Y_{i}-\log \hat{\mu}_{Y \mid I M}\right)^{2}}{N-2}}
$$

where $Y$ represents the critical demand to capacity ratio and $a$ and $b$ are the two linear regression coefficients in the logarithmic scale (similarly a power-law curve can be adopted to fit the data in the original scale). $\hat{\mu}_{Y \mid I M}$ represents the median for $Y$ given a seismic intensity value $I M$, while $\hat{\beta}_{\log Y \mid I M}$ 
is the logarithmic standard deviation for $Y$ conditioned to the $I M$. Finally, the system fragility curve can be obtained as:

$$
P(\log Y>0 \mid I M)=\Phi\left(\frac{\log \hat{\mu}_{Y \mid I M}}{\hat{\beta}_{\log Y \mid I M}}\right)
$$

\section{Proposed non-parametric approximation}

The proposed approach aims at reducing the computational effort by providing a non-parametric approximated solution to compute conditional reliability measures. For the sake of clarity, small letters are used to indicate parameters while capital letters refer to random variables or subsets of the sample space.

Given a failure event $F$ and a sequence of subsets $B_{i}=\left\{\vec{M}, \vec{R} \mid i m_{i}=\right.$ constant $\}$ in the 2-dimensional sample space [moment magnitude - $M$, epicentral distance - $R$ ], the failure probability for a given $B_{i}$ can be expressed following the Kolmogorov definition as:

$$
P\left(F \mid B_{i}\right)=\frac{P\left(F \cap B_{i}\right)}{P\left(B_{i}\right)}
$$

where $P\left(F \cap B_{i}\right)$ represents the joint probability of the events $F$ and $B_{i}$. Moreover, for any failure event $F$, the associated probability of occurrence can be computed as:

$$
P(F)=P_{f}=\int_{F} \Phi(\vec{M}, \vec{R}) d x=\int_{[M, R]} \Phi(\vec{M}, \vec{R}) \cdot \mathbb{I} d x
$$

where $\mathbb{I}$ is an indicator function equal to 1 when the system fails and 0 otherwise, while $\Phi(\cdot)$ represents the joint probability density function in the standard normal space [29]. The mapping from the physical space to the standard normal space can be easily obtained by assuming that the cumulative density functions of the random variables $(M$ and $R$ ) remain the same after the transformation in standard normal distributions [30].

The multi-dimensional integration over the failure domain $F$ is often impossible to perform in a closed form [31]. Hence a simulation-based approach, that approximates the exact solution, is required.

The joint probability $P\left(F \cap B_{i}\right)$, according to Equation (9), can be computed as:

$$
P\left(F \cap B_{i}\right)=\int_{F \cap B_{i}} \Phi(\vec{M}, \vec{R}) d x=\int_{B_{i}} \Phi(\vec{M}, \vec{R}) \cdot \mathbb{I} d x
$$


while the probability $P\left(B_{i}\right)$ in Equation (8) can be computed through the integral of $\Phi(\cdot)$ over $B_{i}$. Equation (8) can be now rewritten as:

$$
P\left(F \mid B_{i}\right)=\frac{P\left(F \cap B_{i}\right)}{P\left(B_{i}\right)}=\frac{\int_{B_{i}} \Phi(\vec{M}, \vec{R}) \cdot \mathbb{I} d x}{\int_{B_{i}} \Phi(\vec{M}, \vec{R}) d x}
$$

An approximated solution of $\int_{B_{i}} \Phi(\vec{M}, \vec{R}) d x$ can be easily reached without additional computational efforts by means of a simulation-based approach, namely by sampling in the standard normal space along each event $B_{i}$. In general, to compute the failure probability conditioned to the value assumed by a specific input, a sequence of samples must be generated in all the subsets of the sample space characterized by a constant value for the input of interest. An efficient solution of Equation (11) still needs an additional procedure to reduce the number of model realizations related to the indicator function $\mathbb{I}$ and thus, to the failure region identification.

\subsection{Failure region mapping}

A Multinomial Logistic Regression (MLR) [32] is adopted to predict the probability that a specific structural system reaches the failure region or not for a given $I M$. The chosen classification algorithm allows the definition of intermediate classes in case conditional failure probabilities with respect other final performance levels are required (i.e. multiple failure regions). The indicator function II in Equation (9) is therefore replaced by the probabilistic score $w_{i}$ that expresses the confidence level of each prevision $Y_{i}$. In particular, the probability that $Y_{i}$ belongs to the class $K$ can be calculated as:

$$
P\left(Y_{i}=K\right)=w_{i}=\frac{e^{\beta_{K} \cdot\left[\vec{M}_{i}, \vec{R}_{i}\right]}}{1+\sum_{k=1}^{K} e^{\beta_{k} \cdot\left[\vec{M}_{i}, \vec{R}_{i}\right]}}
$$

where $\beta_{K}$ represents the vector of regression coefficients associated with the class $K$. Two classes are defined for each failure criterion (Failure or NotFailure) and, if required, multi-dimensional damage measures [33] can be adopted by increasing the number of attributes describing each observation $i$. In case of synthetic ground motion records (stochastic earthquake model), the initial seismic dataset for the $M L R$ training can be obtained by means of a Design of Experiments ( $D o E$ ) simulation based on surrogate probability density functions $(P D F)$ of $M$ and $R$, in order to easily reach the failure 
region getting, at the same time, a higher grade of coverage of the sample space (Table 4). In particular, deterministic samples are generated from uniform distributions with upper and lower boundaries $(U, L)$ chosen according to the real $P D F s$, so that $F(L)$ and $1-F(U)$ can be considered negligible, with $F(\cdot)$ representing the associated $C D F$.

In absence of a stochastic earthquake model the training of the $M L R$ has to be performed over a dataset of real ground motion records consistent with the probabilistic density functions adopted for $M$ and $R$ and able to cover as much as possible the sample space of interest $[M, R]$. Further details are provided in the next section.

Finally, despite the limitations of the $M L R$ algorithm being a linear classifier based on a separating hyperplane, more complex and general failure region shapes can be still mapped by adopting alternative kernels [34]. In this study a second grade polynomial kernel is employed (this choice will be motivated afterwards).

\subsection{Subsets definition}

An estimation of the solution of Equation (10) can now be obtained by sampling from the surrogate PDFs along each predefined subset $B_{i}$ and by evaluating the probabilistic score $w_{i}$ (see Equation 12) associated with each realization:

$$
P\left(F \cap B_{i}\right) \approx \sum_{B_{i}} \Phi(\vec{M}, \vec{R}) \cdot w_{i}
$$

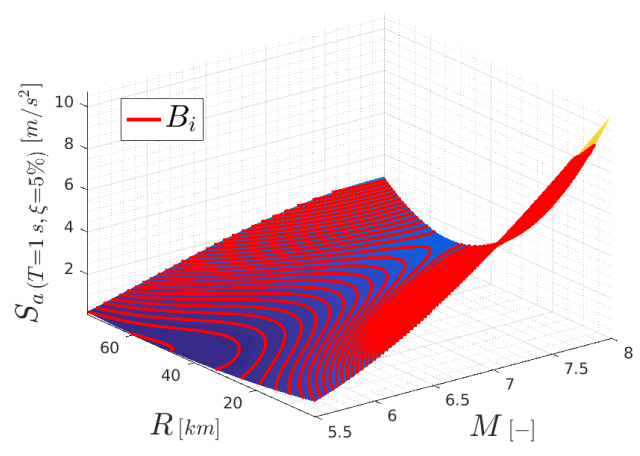

(a)

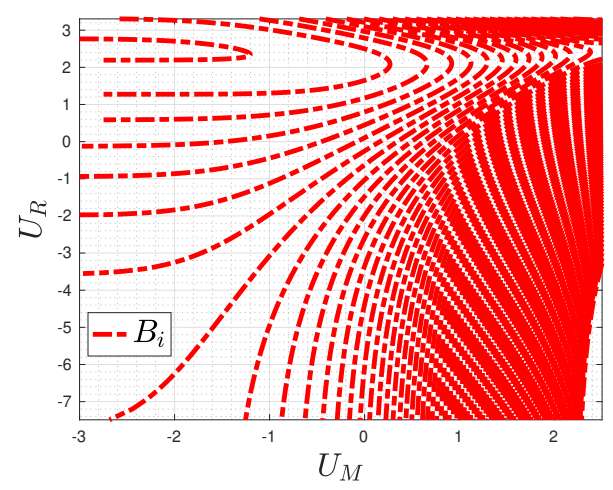

(b)

Figure 1: a) Seismic regression model with a sequence of subsets $B_{i}$, b) Subsets $B_{i}$ in the standard normal space 


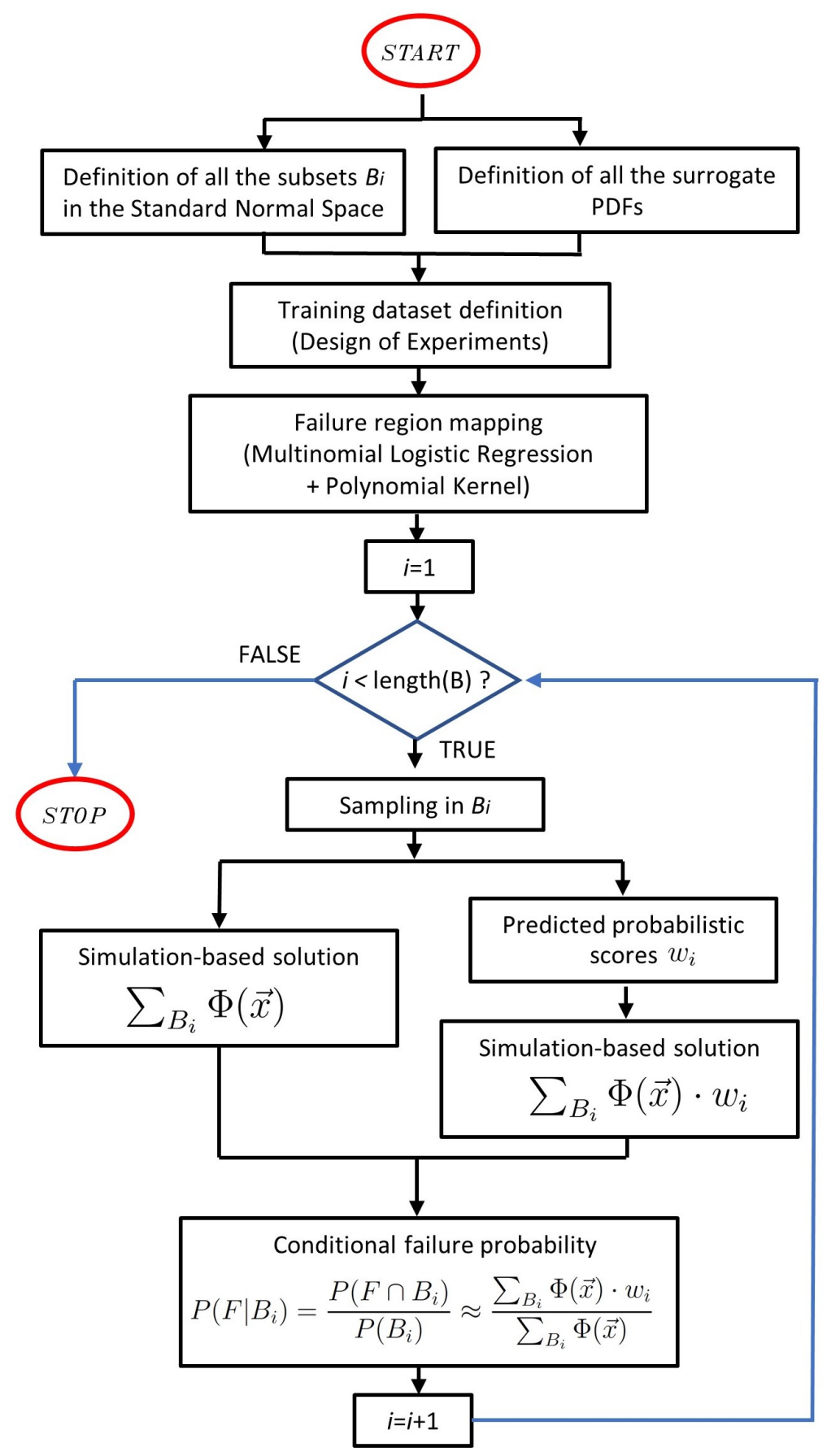

Figure 2: Flowchart describing the proposed approach 
In case of seismic fragility curves each subset $B_{i}$ is characterized by a constant $I M$ value. A seismic prediction model $g(M, R)$ is therefore required to identify the coordinates in terms of magnitude and epicentral distance of each $B_{i} \subsetneq[M, R]$. A polynomial regression model is trained over a synthetic ground motion dataset of 5000 seismic samples and adopted as attenuation law. Figure (1a) reports the regression model and a sequence of subsets $B_{i}$ corresponding to discrete values of the spectral acceleration $S a$ (assumed as $I M)$. Finally, after mapping each subset $B_{i}$ in the standard normal space (Figure 1b), the sought solution can be obtained by combining Equations (13) and (11):

$$
P\left(F \mid B_{i}\right)=\frac{P\left(F \cap B_{i}\right)}{P\left(B_{i}\right)} \approx \frac{\sum_{B_{i}} \Phi(\vec{M}, \vec{R}) \cdot w_{i}}{\sum_{B_{i}} \Phi(\vec{M}, \vec{R})}
$$

The samples generated along $B_{i}$, for a simulation-based estimation of $\Phi(\cdot)$, are affected by a regression error $\varepsilon$ assumed to be normal distributed with mean zero and standard deviation provided by the polynomial regression $g(M, R)$. In Figure (2) a flowchart describes step-by-step the algorithm proposed for the estimation of the conditional failure probability corresponding to different $B_{i}$. It can be summarized as follows:

1 Definition of the subset $B_{i}=\left\{\vec{M}, \vec{R} \mid i m_{i}=\right.$ constant $\}$ by means of a proper ground motion prediction model $g(M, R)$.

2 Definition of surrogate uniform distributions for $M$ and $R$.

3 Design of Experiment simulation to generate the $M L R$ training dataset by exploring the sample space $[M, R]$. The output variable is represented by the structural response.

4 Training of the Multinomial Logistic Regression coupled with a polynomial kernel.

5 Sampling along each event $B_{i}$ to compute $\Phi(\cdot)$ and $w_{i}$.

6 For each subset $B_{i}$ compute the corresponding conditional failure probaility by means of Equation 14. 


\section{Seismic demand}

Seismic reliability analyses are based on ground motion records consistent with the local seismic hazard. Despite the seismic record selection strategy is not the focus of this study, two possible approaches for the seismic demand definition are described below, both compatible with the proposed methodology allowing a probabilistic description of the magnitude $M$, the epicentral distance $R$ and the associated seismic intensity measure $I M$.

\subsection{Stochastic earthquake model}

The first alternative involves the adoption of a stochastic earthquake model to simulate the seismic 'record-to-record variability', as developed by Atkinson and Silva for the California region (Appendix A). This option, adopted in the current study, allows to generate an extensive seismic dataset that in turn, allows to compute an 'exact' seismic fragility curve. This curve can be used as target to assess the accuracy of the proposed approach in comparison to the parametric methodologies previously described in Section 2.

The use of synthetic time histories allows the generation of a large seismic dataset needed for the definition of a consistent regression model $g(M, R)$, subsequently employed to correctly identify all the events $B_{i}=\left\{\vec{M}, \vec{R} \mid i m_{i}=\right.$ constant\} (Figure 1). Moreover, a stochastic seismic model can be coupled with a $D o E$ simulation for the generation of the seismic dataset for the $M L R$ calibration, by improving the global efficiency of the method.

It is noted that the probability density functions adopted to define the magnitude and epicentral distance distribution do not affect the final shape of the fragility curves. The same is not true for the adopted regression model that, if poorly calibrated, can reduce the accuracy of the results due to the altered coordinates of all the subsets $B_{i}$.

In Appendix A it can be noted how the random generation of the ground motion time histories depends on only two seismological random variables, namely the moment magnitude and the epicentral distance, in addition to the initial white noise. Therefore, the proposed methodology is built on a two dimensional samples space, by assuming as constant all the remain parameters required by the adopted seismic model. This requires to recalibrate the model in case of different sites of interest. However, the proposed approach can be even generalized by adopting a multidimensional attenuation law able to account for additional sources of uncertainty. 


\subsection{Real ground motion records}

Three of the basic steps that compose a classic Probabilistic Seismic Hazard Analysis (PSHA) $[35,36]$ are necessary to couple the proposed approach with real ground motion records.

1 The definition of a probability density function describing the occurrence of the earthquake magnitudes $M$.

2 A probabilistic description of the earthquake epicentral distances $R$ based on a previous identification of the earthquake source type (e.g. line source, area source).

3 The ground motion intensity measure $I M$ has to be predicted starting from $M$ and $R$. This can be done by means of existing ground motion prediction models that are generally based and calibrated on statistical regressions over a large dataset of observed seismic intensity measures.

All the subsets $B_{i}$ can then be defined on the basis of the adopted attenuation law, while the probability density functions of $M$ and $R$ allow computing $\Phi(\cdot)$ in the standard normal space. The dataset of real time histories can finally be used for the $M L R$ training. It is worth pointing out, however, that the use of a real dataset will reduce the efficiency of the approach since advanced sampling techniques can not be adopted for the definition of the training dataset.

\section{Case studies}

Two different structural systems are analyzed to test and validate the proposed approach. Both models do not require an excessive computational effort in order to derive the target seismic fragility curve through a Latin Hypercube simulation. The opensource Matlab toolbox OpenCossan [37, 38] is used for the probabilistic analysis.

\subsection{Impact model}

A dissipative Kelvin-Voight impact model [39], improved by Komodromos in 2007 [40], is added to a single-degree-of-freedom system (Figure 3) in order to analyze the collisions between the superstructure and the lateral walls [41]. The magnitude of the impact force during the seismic event is computed as 


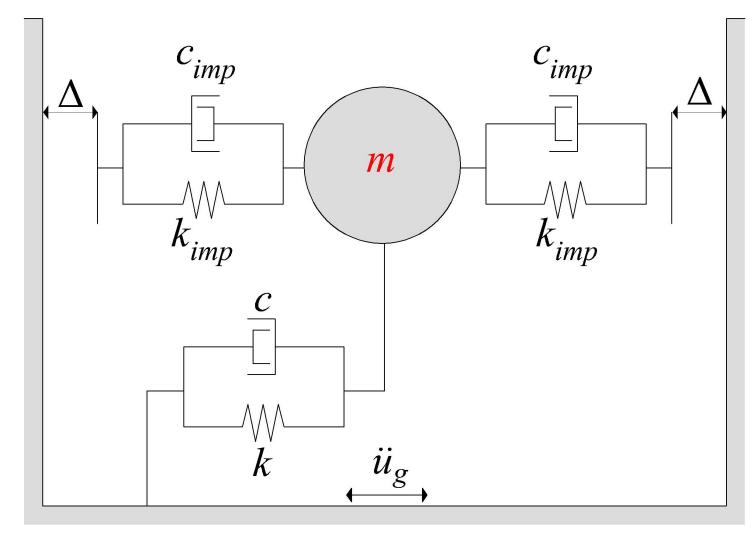

Figure 3: SDOF impact model

follows:

$$
\begin{gathered}
F_{i m p}(t+\Delta t)=k_{i m p} \cdot \delta(t)+c_{i m p} \cdot \dot{\delta}(t) \text { when } F_{i m p}(t)>0 \\
F_{i m p}(t+\Delta t)=0 \text { when } F_{i m p}(t) \leq 0
\end{gathered}
$$

where $\delta(t)$ and $\dot{\delta}(t)$ represents the interpenetration depth and the relative velocity, respectively, computed in Matlab by means of an explicit RungeKutta method [42] and a time step of $10^{-5}$ seconds. $k_{i m p}$ is the impact stiffness while $c_{i m p}$ the impact damping coefficient [40]. Further details on the model characterization can be found in [41]. Table 1 reports the structural parameters adopted for the dynamic analysis, in particular a reduced $\Delta$ is employed in order to obtain a probability of having impacts almost equal to 1 .

\begin{tabular}{cccc}
\hline $\mathbf{m}[\mathbf{t o n}]$ & $k_{i m p} / k$ & $\mathbf{T}[\mathbf{s}]$ & $\Delta[\mathbf{m}]$ \\
\hline 100 & 10 & 1 & 0.001 \\
\hline
\end{tabular}

Table 1: Adopted structural configuration for the SDOF impact model

\subsection{Viscous Dampers}

In the second case study the seismic reliability of a steel moment-resisting frame equipped with non linear viscous dampers is analyzed (Figure 4). The 
structure has been already employed in several studies for retrofit applications ( e.g. [43, 44, 45]) and represents typical low-rise buildings of the Los Angeles, California region [43]. The dynamic analysis of the 2D model shown in Figure (4) is performed by the opensource FEM software OpenSees [46]. The vibration periods associated to the first 3 modes are $0.99 \mathrm{~s}, 0.33 \mathrm{~s}$ and 0.18 s respectively, while the total mass corresponding to the two lower levels is equal to 975 tons and it reaches 1040 tons at the top floor.

Columns and beams are both wide flanges with section dimensions shown in Figure 4. A P-Delta column is employed within the model to account for the nonlinear geometrical effects due to the action of relevant vertical loads and a corotational transformation is employed to capture the large displacementsmall strain effects. Finally, distributed plasticity is used to model nonlinear elements and fiber sections. Additional details on the FEM model and the structural properties can be found in [47, 43].
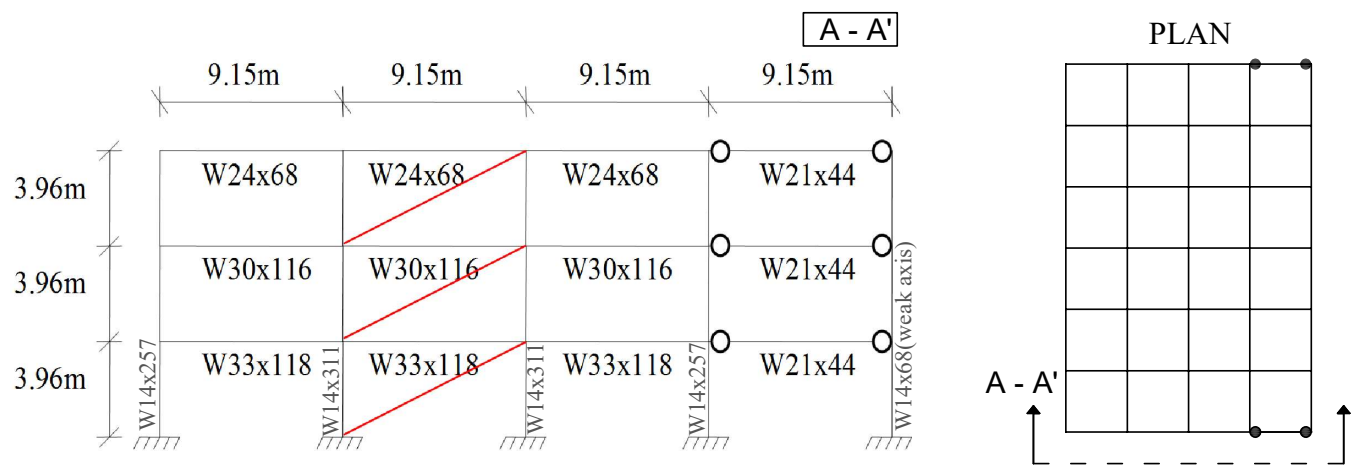

Figure 4: Steel moment-resisting frame equipped with non linear viscous dampers

The damping force can be modeled by means of the following constitutive law:

$$
F_{d}(\dot{u})=C_{d} \cdot|\dot{u}|^{\alpha} \cdot \operatorname{sign}(\dot{u})
$$

where $C_{d}$ represents the damping constant, $\dot{u}$ is the relative velocity between the damper extremities and finally the exponent $\alpha$ controls the nonlinearity of the damper response. Seismic fragility curves are derived for the non linear case with $\alpha=0.3$. 


\subsection{Results}

The probabilistic analysis is carried out by considering three increasing structural reliability levels associated with different dynamic performance required. Table 2 reports the adopted thresholds for each case study, in terms of inter-storey drift $(I D R)$ and impact force $(I F)$. It is worth noting that the performance levels required to the steel moment resisting frame might appear too low, however the presence of non linear viscous damper significantly increase the dissipative capacity of the original structure and an IDR $=0.6 \%$ already generates a sufficiently rare failure region.

\begin{tabular}{cccc}
\hline Impact model & Level 1 & Level 2 & Level 3 \\
IF & $200 \mathrm{kN}$ & $400 \mathrm{kN}$ & $600 \mathrm{kN}$ \\
\hline Viscous dampers & Level 1 & Level 2 & Level 3 \\
IDR & $0.1 \%$ & $0.3 \%$ & $0.6 \%$ \\
\hline
\end{tabular}

Table 2: Performance levels analyzed for each case study

\subsubsection{Parametric approaches}

A seismic dataset of 90 time histories is generated through a $L H S$ by sampling $M$ and $R$ (Figure 9b,d) from the original distributions (Appendix A) and then employed to perform both the $C A$ and $I D A$. The dataset defined for the proposed method (Figure 5), obtained by a $9^{2}$ full factorial $D o E$, cannot be used for the IDA since the surrogate $P D F_{s}$ would strongly affect the final distribution of the $I M_{s}$ corresponding to the failure state, leading to inaccurate results.

A ground motion dataset based on 30 incremental values of $S a$ is defined for the $M S A$. In particular, 30 accelerograms have been selected for each considered $I M$ level, reaching a total of 900 ground motion time histories. All the intermediate datasets of 30 samples are obtained through one single Subset Simulation analysis (SS) [48], able to efficiently direct the samples generation towards higher $I M$ levels by mean of Markov chain Monte Carlo. It is worth highlighting that the number of stripes can be further optimized to reduce the seismic dataset required by the method. Indeed, the final efficiency and accuracy are also dependent on the amplitude of each stripe, as shown in Eads et al. [49]. However, to reach a priori a global optimal calibration of the $M S A$, its is required a more in depth knowledge on the system performances that, most of the times, is impossible to obtain. 
In order to assess the performances of the proposed method in terms of both accuracy and efficiency, the analyzed parametric approaches have to be performed with a similar number of ground motion time histories to get final comparable results. However, the $M S A$, being based on multiple statistical analysis corresponding to increasing $I M$ levels, requires a higher number of seismic samples comparing to the other approaches.

Moreover, similarly to the proposed approach that is performed always on the same initial seismic dataset (regardless the specific case study or the required performance level), the datasets for the parametric approaches are purposely not calibrated according to the different analyzed case. Finally, it worths noting that the proposed approach is the one performed with the lowest number of seismic samples since it can be coupled with a $D o E$ simulation to efficiently cover the sample space in case of artificial time histories.

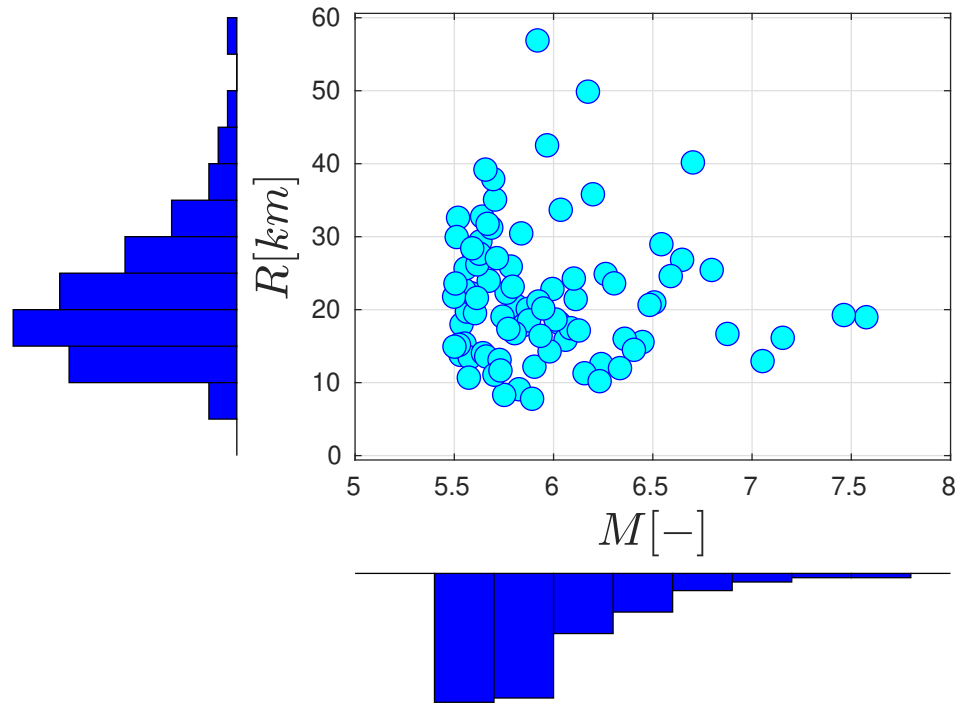

Figure 5: $M$ and $R$ samples employed for the $C A$ and the $I D A$.

For the $C A$, a probabilistic demand model is defined by means of a second grade polynomial regression that turned out to be more accurate than a simple linear model (Figure 6). 


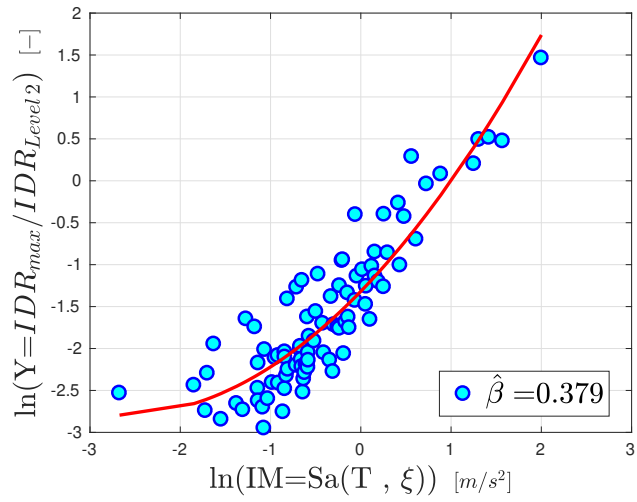

(a)

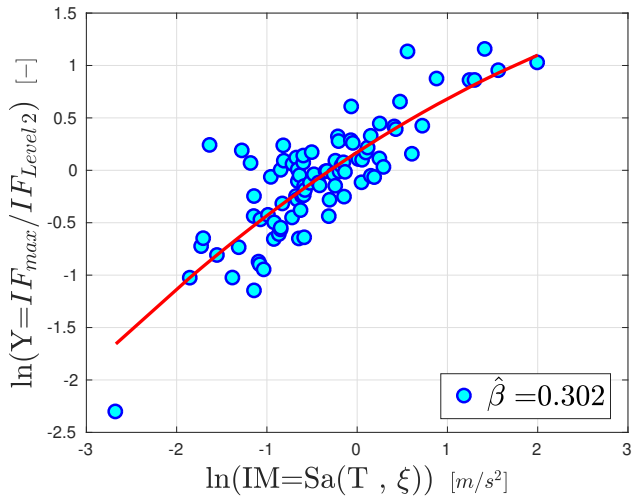

(b)

Figure 6: Probabilistic demand model employed by the Cloud Analysis for a) the steel frame equipped with viscous dampers and b) the SDOF impact model. Final structural threshold based on the performance level 2 .

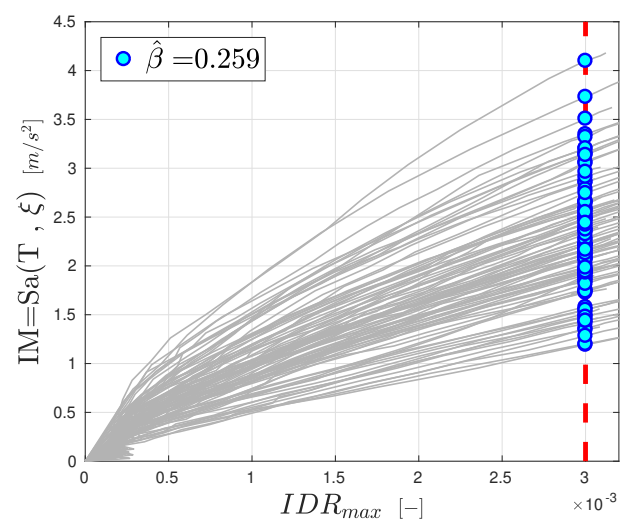

(a)

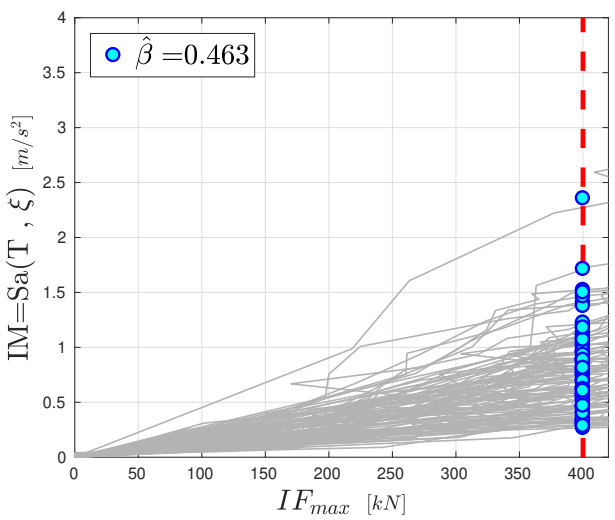

(b)

Figure 7: IDA curves for a) the steel frame equipped with viscous dampers and b) the SDOF impact model. Final structural threshold based on the performance level 2. 


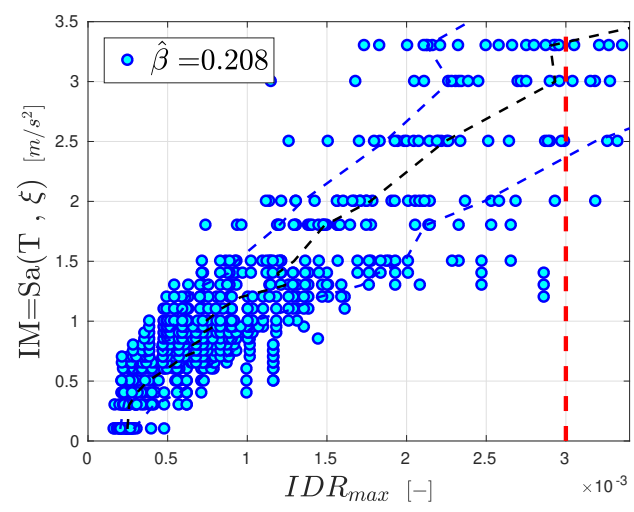

(a)

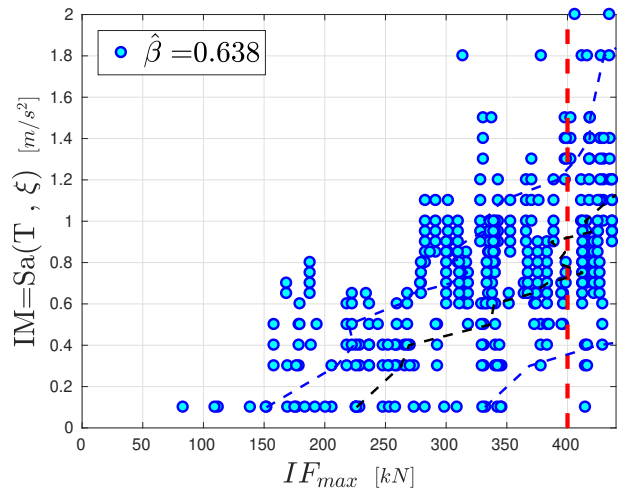

(b)

Figure 8: Structural samples for each IM value considered in the MSA for a) the steel frame equipped with viscous dampers and b) the SDOF impact model. Final structural threshold based on the performance level 2. The median of each stripe is reported together with the 84th and 16th percentiles

The IDA is performed by adopting a more efficient algorithm [11] able to adaptively scale the initial ground motion by respecting a tolerance of $5 \%$ on the final interpolated $I M$ value corresponding to the failure state. Figure (7) shows the $90 I D A$ curves for the two case studies, assuming Level 2 as final threshold.

Figure (8) reports the structural responses associated with each $I M$ value considered in the MSA, the median response with the 84th and 16th percentiles are shown as well. Table 3 summarizes the employed seismic datasets together with the associated sampling technique.

\begin{tabular}{ccc}
\hline Methodology & Seismic dataset dimension & Sampling Technique \\
\hline Method Proposed & 81 & DoE \\
$C A$ & 90 & $L H S$ \\
$I D A$ & 90 & $L H S$ \\
$M S A$ & $900(30 \times 30)$ & $S S$ \\
\hline
\end{tabular}

Table 3: Seismic datasets

\subsubsection{Failure region mapping}

The failure region mapping, required to compute the probabilistic scores $w_{i}$, is performed by adopting a $M L R$ coupled with a second grade polynomial 


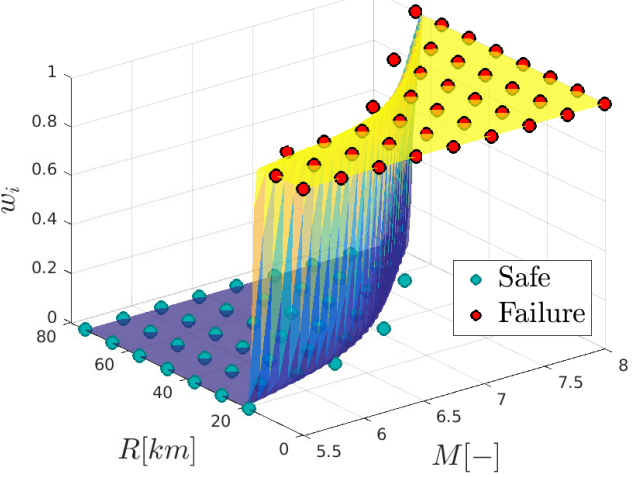

(a)

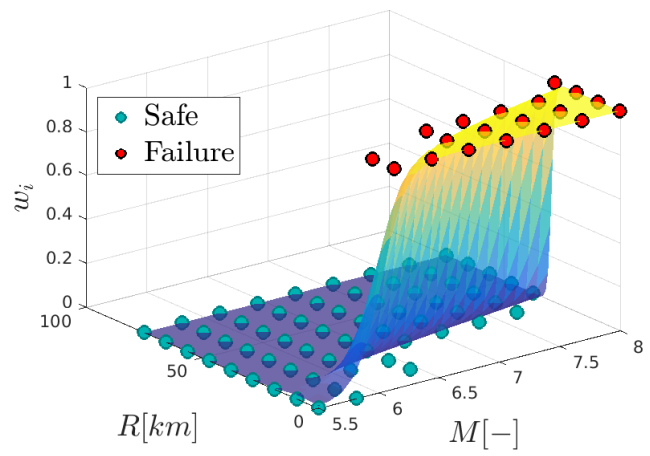

(c)

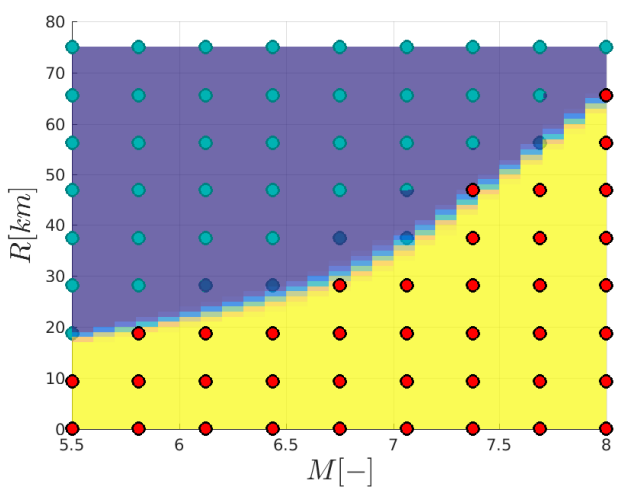

(b)

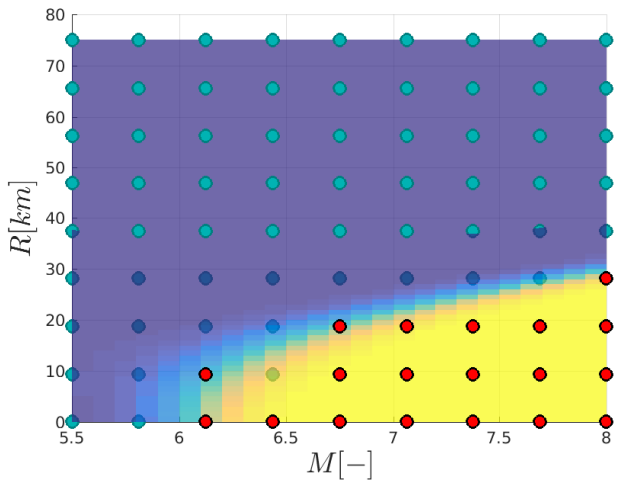

(d)

Figure 9: Multinomial logistic regression with failed and safe structural samples for a) the impact model and c) the viscous dampers. Sub-figures b) and c) show the efficiency of the adopted quadratic polynomial kernel (Level 2).

The optimal grade of the polynomial kernel depends on the shape of the failure region, for the analyzed case studies a second grade polynomial is 
an acceptable compromise between the classification accuracy and the computational costs. Moreover, a higher polynomial grade associated with a reduced training dataset may increase the probability of having overfitting. For complex failure region shapes more performing kernels can be employed (e.g. Radial basis function kernel). Figures (9a) and (9c) show the trained logistic regression for two specific cases with the associated training dataset (81 structural responses) obtained through a $9^{2}$ Full Factorial DoE simulation. The surrogate PDFs, employed for the training dataset definition, are reported in Table 4. From Figures (9b) and (9d) it can observed how the $M L R$ is able to perform a non-linear classification thanks to the adopted kernel. The employed multiclass classification algorithm allows to get multiple fragility curves for different structural thresholds after one single training stage. Though Figure (9b) shows an almost clean transition from the safe to the failure region, this depends on the number of levels adopted for the $D o E$ simulation. In fact, the noise associated to the transition appears more visible by increasing the number of samples within the sample space (Figure 10). Moreover, it should be noted that an additional level of uncertainty comes from the $S a$ associated to each couple $(M, R)$, that means we can experience failure or not-failure even with the same $M$ and $R$, especially around the transition zone.

The $M L R$ training is affected by the dimension of the initial seismic dataset and the same applies to the failure region shape. Figure (9d) is therefore a direct consequence of the reduced number of time histories employed by the proposed method. The adopted sparse grid of seismic samples (Figure 10a) leads to a net separation between safe and failure samples and this is reflected in the mapping process. The method, however, is able to moderate the effects of this approximation by computing the probabilistic scores $w_{i}$ over the whole subset $B_{i}$ (Equation 14), and so, by overestimating and underestimating at the same time the failure probability around the edges of the failure region. 


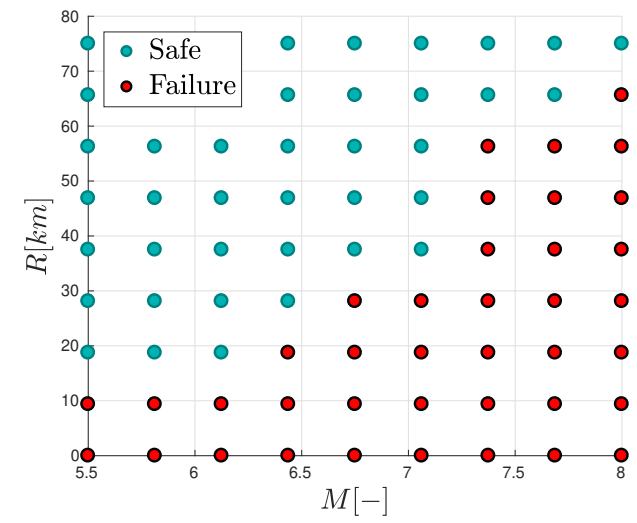

(a)

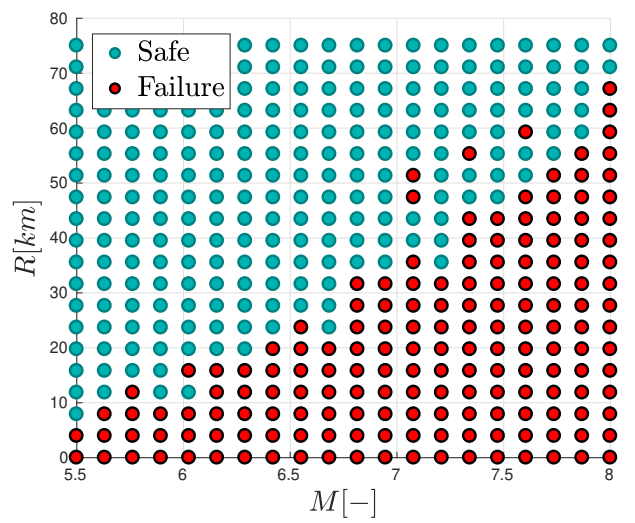

(b)

Figure 10: $9^{2}$ a) and $20^{2}$ b) Full Factorial DoE for the viscous dampers model (Level 2).

\begin{tabular}{cccc}
\hline Quantity & Surrogate PDF & L & U \\
\hline$M$ & Uniform & 5.5 & 8 \\
$R$ & Uniform & $0 \mathrm{~km}$ & $75 \mathrm{~km}$ \\
\hline
\end{tabular}

Table 4: Surrogate uniform distributions of $M$ and $r$

\subsubsection{Fragility curves}

The seismic fragility curves obtained by means of parametric approaches are compared with the results provided by the proposed procedure (Figures 12 and 13). Moreover, a target solution is computed with 20000 structural dynamic analyses based on as many synthetic ground motion records generated by sampling $\mathrm{M}$ and $\mathrm{R}$ through a $L H S$. In particular, a discrete number of finite $S_{a}$ intervals (delimited by the vertical lines in Figure 11) have been identified and for each of them the conditional failure probability is computed as the ratio between the number of samples above the structural threshold of interest (red lines in Figure 11) and the total number of sample in the same $S_{a}$ interval. The width of the $S a$ intervals is assumed constant and it has been chosen by increasing as much as possible the final number of considered intervals, ensuring at the same time a sufficient number of samples falling in each of them. 


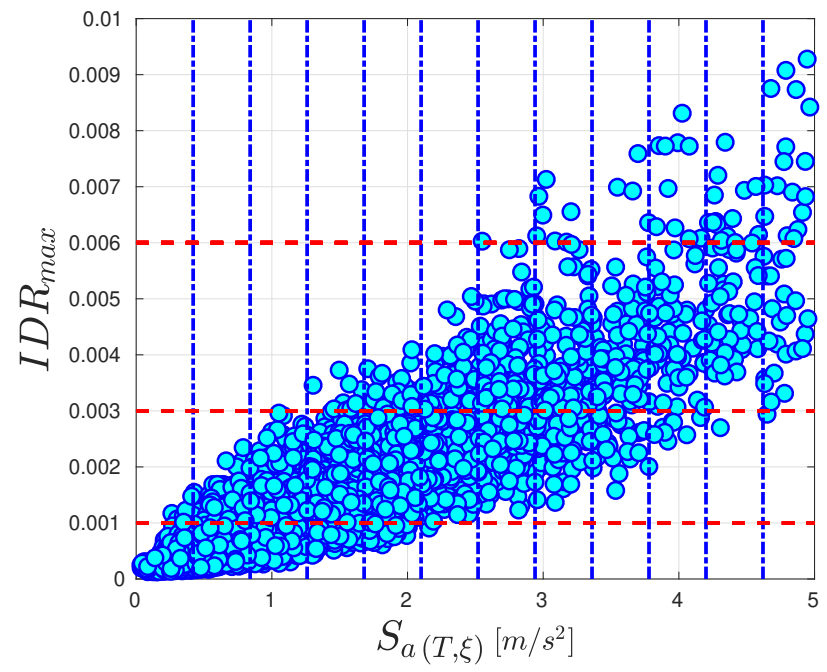

Figure 11: LHS samples for the target fragility curve estimation with the three performance levels of interest and the finite intervals of $S_{a}$ (viscous dampers).

The target conditional failure probability is estimated for a finite number of intervals of the seismic intensity measure $(S a)$. For each considered interval $j$, the estimated target value is reported together with the standard deviation of the seismic samples in $j$ and the associated confidence bounds computed as the standard deviation of a Bernoulli distribution (failure/safe). In addition to Figures 12 and 13, a comparison in terms of Mean Squared Error $(M S E)$ computed with respect to the target curve is provided in Figure 14.

Analyzing the results, the following are the most relevant aspects to highlight:

1. The proposed methodology returns seismic fragility curves with the best overall behavior if compared with the LHS target solution and the parametric approaches.

2. For the viscous dampers case study, the parametric methodologies tend to perform poorly in case of high structural thresholds, that are exceeded for seismic intensity measures characterized by a low probability of occurrence (Figure 12c). This trend is less clear for the impact model, since the performance levels considered are less demanding, generating larger failure regions (Figure 9b).

3. Thus, for failure regions reached only by rare seismic intensity levels, the Cloud Analysis needs to increase the employed dataset for the demand model definition while the Incremental Dynamic Analysis tends 
to lose accuracy due to the scaling process.

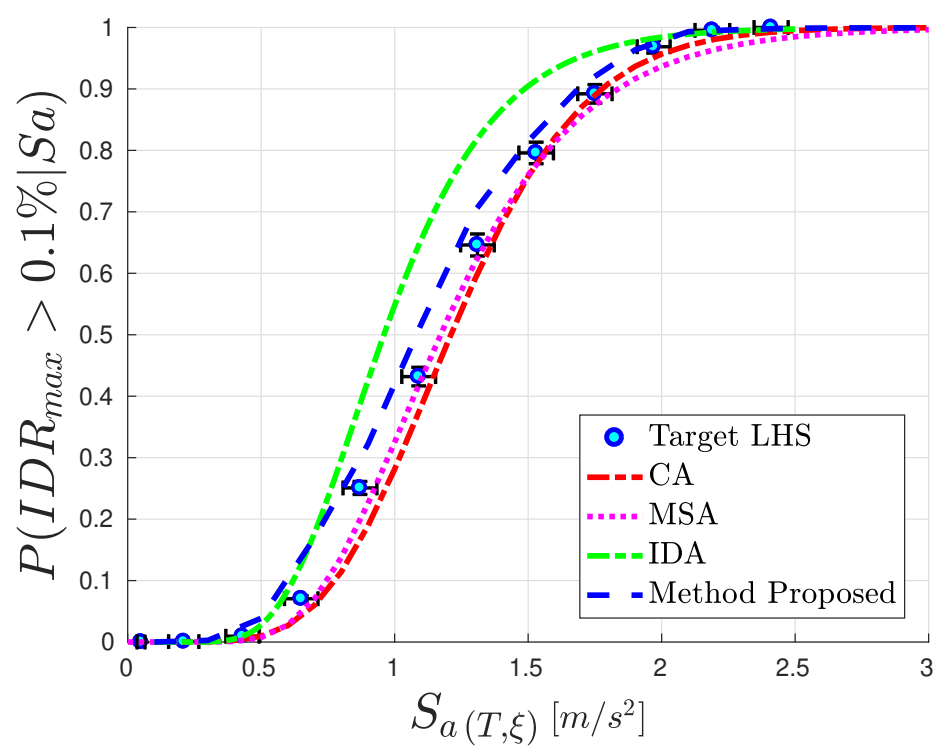

(a)

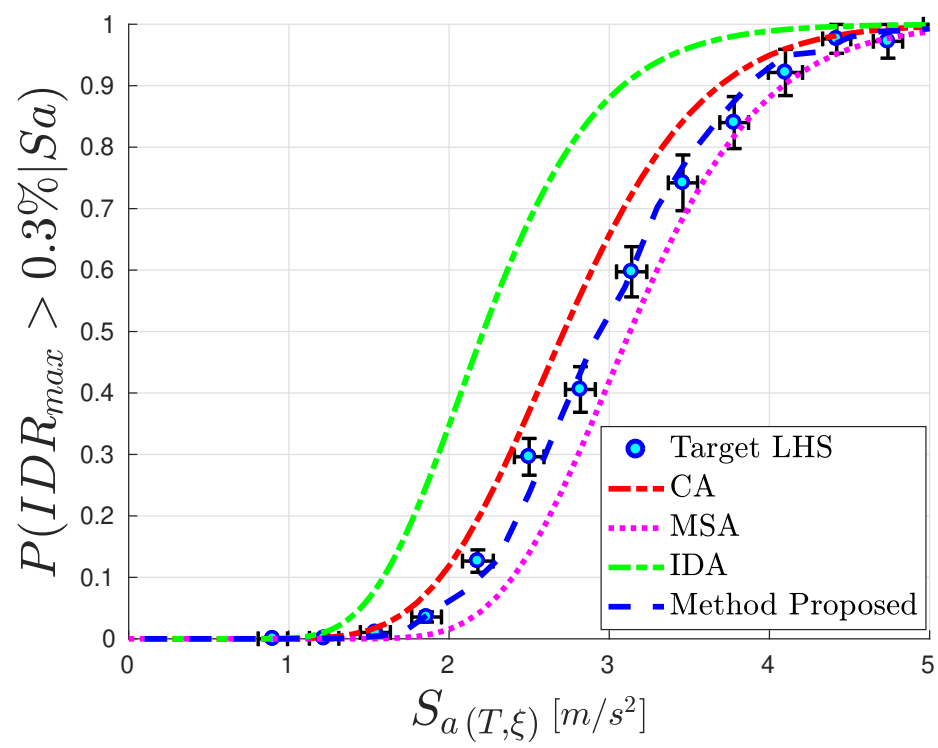

(b) 


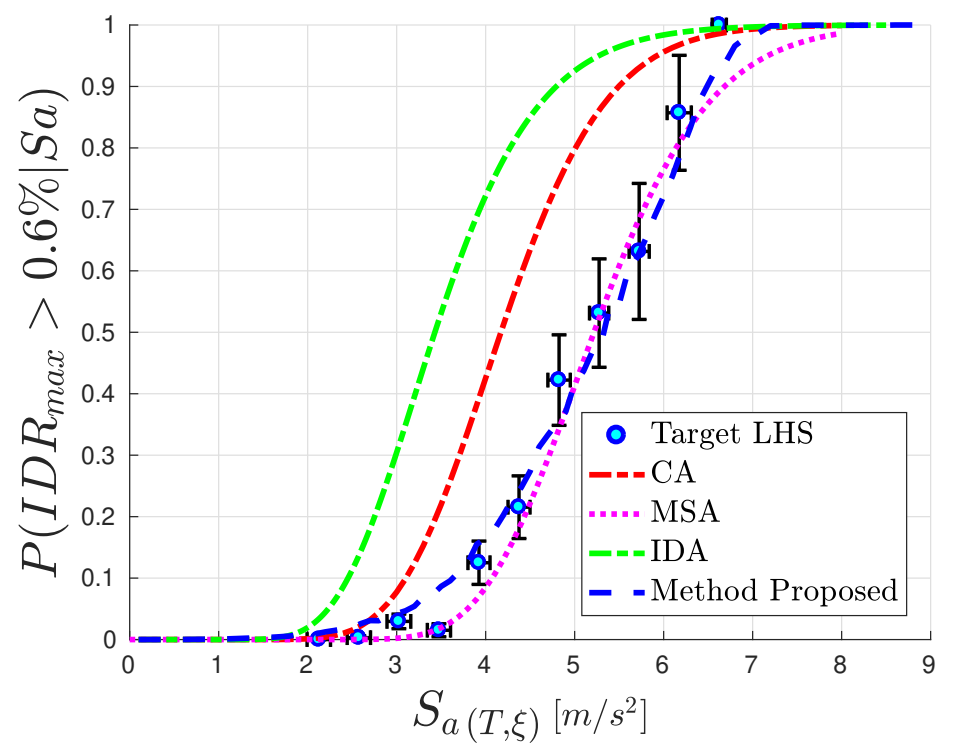

(c)

Figure 12: Comparison between the parametric approaches, the proposed method and the target solution for the steel moment resisting frame. The result are reported for a) Level 1, b) Level 2 and c) Level 3 of the final structural threshold.

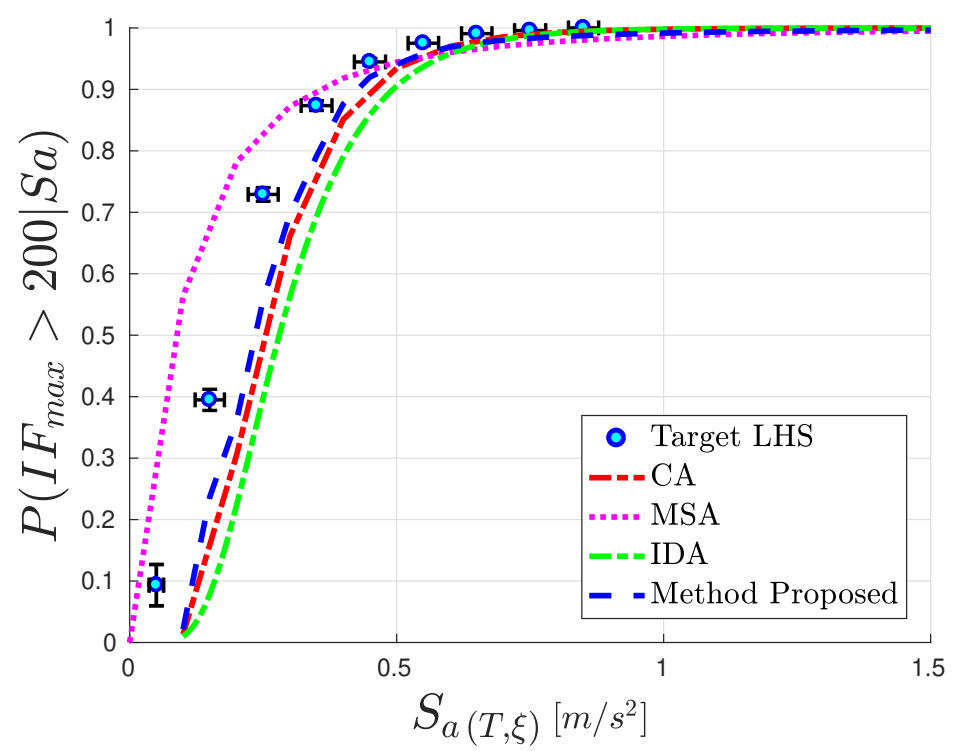

(a) 


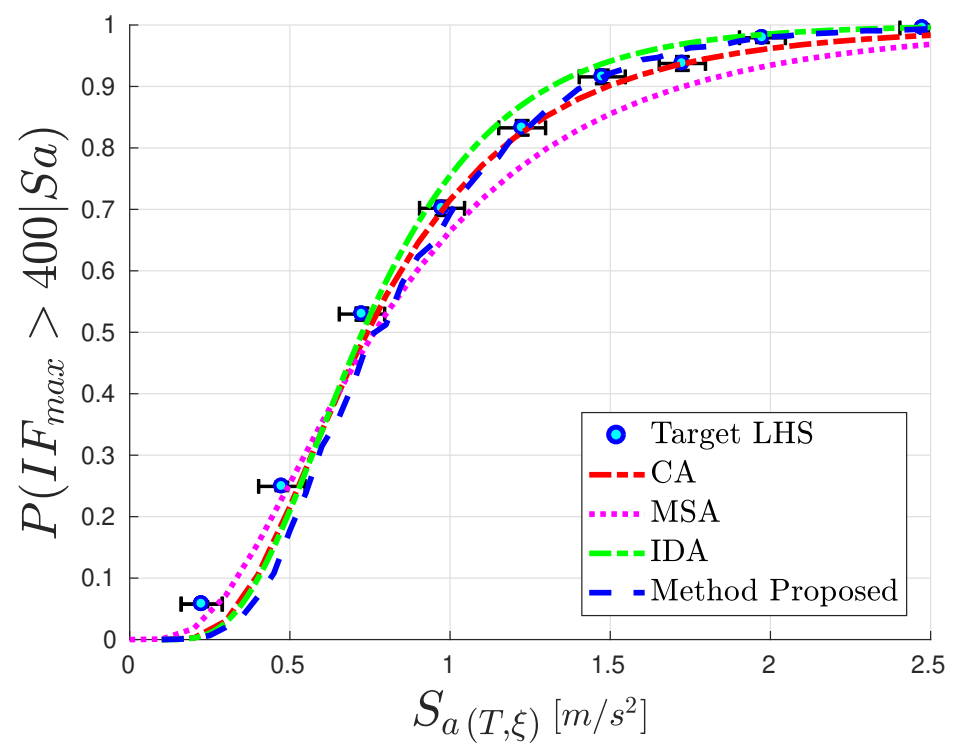

(b)

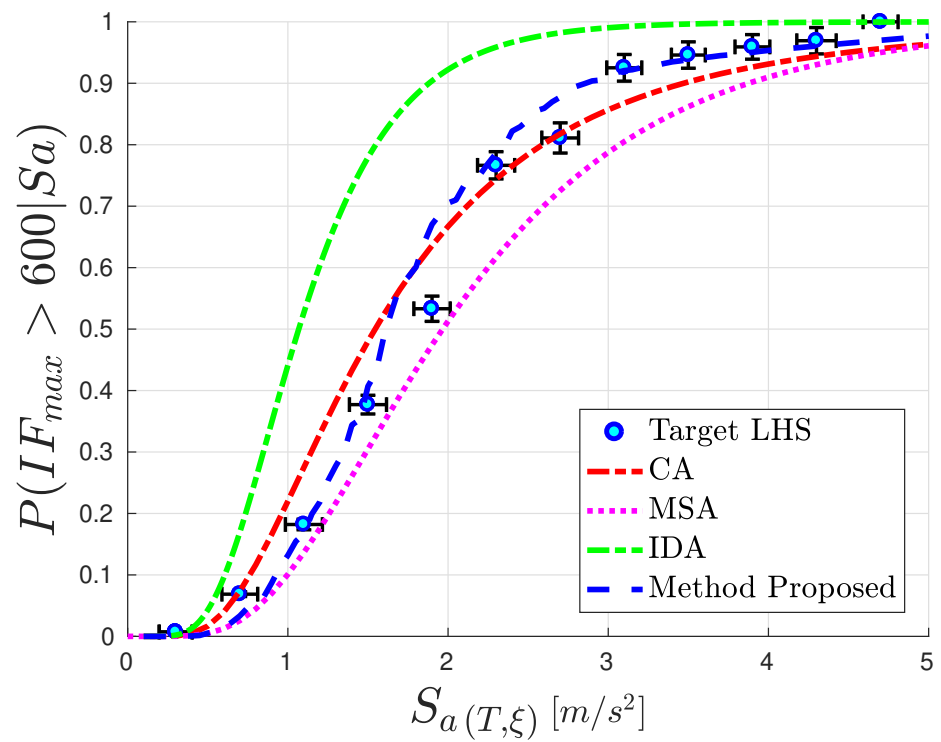

(c)

Figure 13: Comparison between the parametric approaches, the proposed method and the target solution for the SDOF impact model. The result are reported for a) Level 1, b) Level 2 and c) Level 3 of the final structural threshold. 


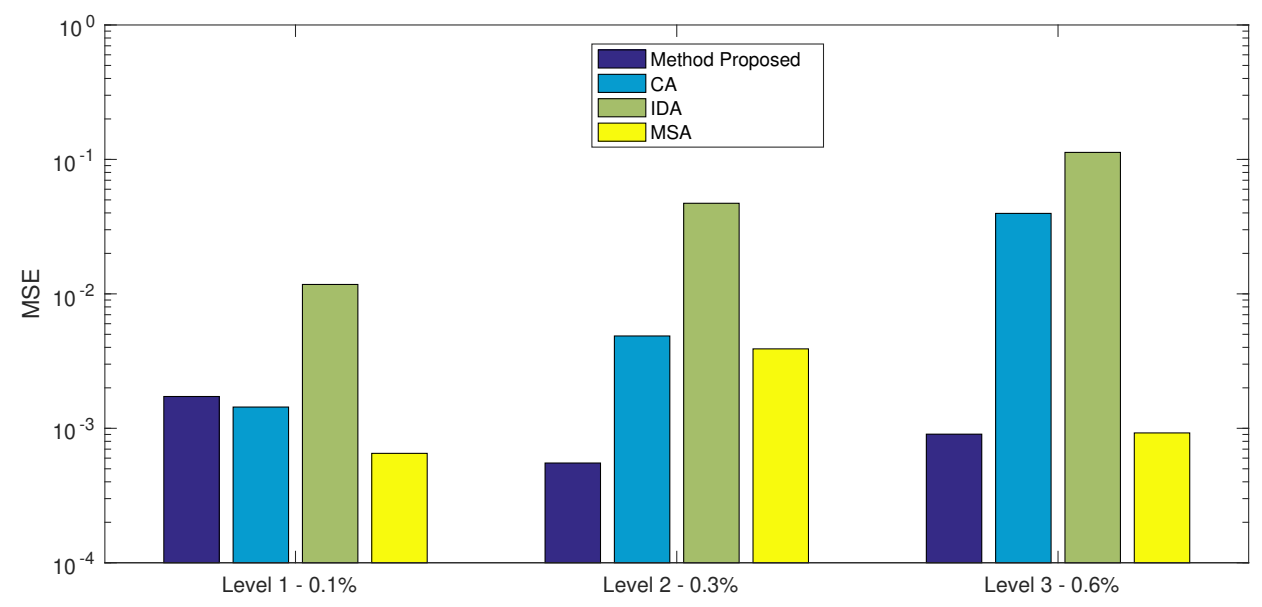

(d)

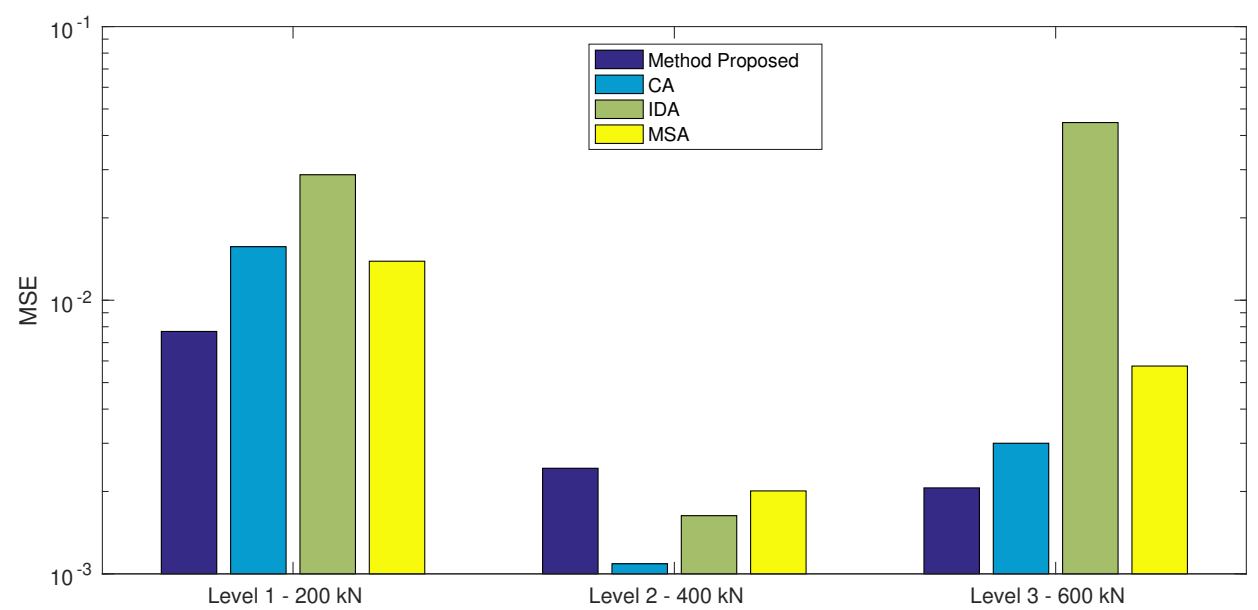

(e)

Figure 14: Mean Squared Error between the target fragility curve and the different analyzed approaches for a) the viscous dampers case study and b) the impact model.

The Multiple Stripe Analysis can exploit an initial dataset 10 times larger than the other parametric approaches, obtaining more stable performances in almost every analyzed case.

The proposed approach appears to be more reliable and efficient since all the considered parametric methodologies share an increase in terms 
of samples required in case of rare failure events (e.g. Figure 9d).

4. Among the analyzed parametric procedures, the Cloud Analysis shows a higher accuracy for the impact model case study and it results slightly more accurate than the proposed method in 2 cases out of the 6 analyzed. The Incremental Dynamic Analysis shows problems for highperformance levels and this may be caused by the initial seismic dataset that needs to be heavily scaled in order to reach the failure region [17]. Finally, the Multiple Stripe Analysis thanks to the extended initial seismic dataset returns stable performances, providing the best fragility curve in only 1 case.

It worths noting that the analyzed parametric approaches have shown reliable performances in many other studies (e.g. [21, 50, 51, 52]). The structural models analyzed in the present work result more demanding and tricky due to the high nonlinearity of the response domain, but this is expected due the difficulty to efficiently populate the region of the sample space corresponding to high $S_{a}$ values and multiple system failures [16]. In general, the accuracy of parametric fragility curves can therefore be more sensitive to the structural typology, in addition to the adopted EDP, IM and initial seismic dataset [11].

\section{Conclusions}

An efficient non-parametric methodology to compute seismic fragility curves has been proposed. A stochastic earthquake model is employed to generate the ground motion dataset . The failure region mapping process based on the use of surrogate PDFs and a Multinomial Logistic Regression allows decreasing the number of samples required. The method is able to manage noise along the failure region boundaries thanks to the adoption of probabilistic scores $w_{i}$ as indicator function, while more complex failure regions can still be mapped by the adopted kernel. The non-parametric approach does not assume any predefined shape for the final fragility curve, preventing a possible lack of fitting and additional drawbacks characterizing the analyzed parametric methodologies.

The proposed approach returns the most accurate results for almost every performance level and case study considered. This appears even more evident in case of failure regions characterized by a low probability of occurrence where parametric approaches require an increase of computational cost 
507 and/or initial seismic dataset dimensions.

508

509

510 Acknowledgements

511 The authors would like to acknowledge the gracious support of this work 512 through the EPSRC and ESRC Centre for Doctoral Training on Quantifi${ }_{513}$ cation and Management of Risk and Uncertainty in Complex Systems and 514 Environments Grant number (EP/L015927/1).

515 


\section{Appendix A: Stochastic seismic model}

The model is completely defined by the ground motion's radiation spectrum $A(f ; M, R)$ and its time envelope function $e(t ; M, R)$, both depending on the magnitude $M$ and the epicentral distance $R$ and defined as:

$$
\begin{gathered}
A(f ; M, R)=A_{0}(f) \cdot V(f) \cdot 1 / r \cdot \exp [-\gamma(f) \cdot R] \cdot \exp (-\pi f k) \\
e(t ; M, R)=c_{3} \cdot t^{c_{1}} \cdot \exp \left(-c_{2} \cdot t\right) \cdot U(t)
\end{gathered}
$$

In the Equation (18), $A_{0}$ is the 'equivalent point-source spectrum', $V(f)$ describes the amplifications through the crustal velocity gradient, $1 / r$ is the geometric spreading factor, $\gamma(f)$ represents the anelastic attenuation and finally the term $\exp (-\pi f k)$ simulates the near surface attenuation of highfrequency amplitudes. In the Equation (19), $U(t)$ is a unit-step function, while the expressions of the parameters $c_{1}, c_{2}$ and $c_{3}$ along with all the details to completely derive $A(f ; M, R)$ and consequently the final ground motion sample are described in [53].

The uncertainty associated to $M$ is modeled by adopting the following probability density function, obtained from a truncated Gutenberg-Richter (G-R) relationship (e.g. [54]) in the interval $\left[M_{\min }, M_{\max }\right]=[5.5,8]$ :

$$
p(M)=\frac{b \cdot \exp (-b \cdot M)}{\exp \left(-b \cdot M_{\min }\right)-\exp \left(-b \cdot M_{\max }\right)}
$$

Finally, the uncertainty in the earthquake location is taken into account by assuming $\log (R)$ normally distributed with mean $\log (20)$ and standard deviation 0.5 (e.g. [55]). 
[1] C. Cornell, H. Krawinkler, Progress and challenges in seismic performance assessment cornell. peer center news 3 (2), 2000.

[2] K. A. Porter, An overview of peers performance-based earthquake engineering methodology, in: Proceedings of ninth international conference on applications of statistics and probability in civil engineering, Citeseer (2003).

[3] A. B. Liel, G. G. Deierlein, Assessing the collapse risk of California's existing reinforced concrete frame structures: metrics for seismic safety decisions, volume 69, 2008.

[4] L. F. Ibarra, H. Krawinkler, Global collapse of frame structures under seismic excitations, Pacific Earthquake Engineering Research Center Berkeley, CA, 2005.

[5] D. Straub, A. Der Kiureghian, Improved seismic fragility modeling from empirical data, Structural safety 30 (2008) 320-336.

[6] N. I. Basoz, A. S. Kiremidjian, S. A. King, K. H. Law, Statistical analysis of bridge damage data from the 1994 northridge, ca, earthquake, Earthquake Spectra 15 (1999) 25-54.

[7] B. G. Nielson, Analytical fragility curves for highway bridges in moderate seismic zones, Ph.D. thesis, Georgia Institute of Technology, 2005.

[8] M. Rota, A. Penna, G. Magenes, A methodology for deriving analytical fragility curves for masonry buildings based on stochastic nonlinear analyses, Engineering Structures 32 (2010) 1312-1323.

[9] D. J. Wald, V. Quitoriano, T. H. Heaton, H. Kanamori, C. W. Scrivner, C. B. Worden, Trinet shakemaps: Rapid generation of peak ground motion and intensity maps for earthquakes in southern california, Earthquake Spectra 15 (1999) 537-555.

[10] M. D. Petersen, A. D. Frankel, S. C. Harmsen, C. S. Mueller, K. M. Haller, R. L. Wheeler, R. L. Wesson, Y. Zeng, O. S. Boyd, D. M. Perkins, et al., Documentation for the 2008 update of the United States national seismic hazard maps, Technical Report, Geological Survey (US), 2008. 
[11] D. Vamvatsikos, C. A. Cornell, Incremental dynamic analysis, Earthquake Engineering \& Structural Dynamics 31 (2002) 491-514.

[12] J. W. Baker, Efficient analytical fragility function fitting using dynamic structural analysis, Earthquake Spectra 31 (2015) 579-599.

[13] F. Jalayer, Direct probabilistic seismic anaysis: implementing non-linear dynamic assessments, Stanford University Stanford, 2003.

[14] D. Lallemant, A. Kiremidjian, H. Burton, Statistical procedures for developing earthquake damage fragility curves, Earthquake Engineering \& Structural Dynamics 44 (2015) 1373-1389.

[15] M. Shinozuka, M. Q. Feng, J. Lee, T. Naganuma, Statistical analysis of fragility curves, Journal of engineering mechanics 126 (2000) 1224-1231.

[16] F. Jalayer, H. Ebrahimian, A. Miano, G. Manfredi, H. Sezen, Analytical fragility assessment using unscaled ground motion records, Earthquake Engineering \& Structural Dynamics 46 (2017) 2639-2663.

[17] J. W. Baker, C. Allin Cornell, A vector-valued ground motion intensity measure consisting of spectral acceleration and epsilon, Earthquake Engineering \& Structural Dynamics 34 (2005) 1193-1217.

[18] A. Miano, F. Jalayer, H. Ebrahimian, A. Prota, Cloud to ida: Efficient fragility assessment with limited scaling, Earthquake Engineering \& Structural Dynamics 47 (2018) 1124-1147.

[19] P. Bazzurro, C. A. Cornell, N. Shome, J. E. Carballo, Three proposals for characterizing mdof nonlinear seismic response, Journal of Structural Engineering 124 (1998) 1281-1289.

[20] F. Jalayer, C. Cornell, Alternative non-linear demand estimation methods for probability-based seismic assessments, Earthquake Engineering \& Structural Dynamics 38 (2009) 951-972.

[21] J. W. Baker, Probabilistic structural response assessment using vectorvalued intensity measures, Earthquake Engineering \& Structural Dynamics 36 (2007) 1861-1883. 
[22] H. Y. Noh, D. Lallemant, A. S. Kiremidjian, Development of empirical and analytical fragility functions using kernel smoothing methods, Earthquake Engineering \& Structural Dynamics 44 (2015) 1163-1180.

[23] H. Y. Noh, D. G. Lignos, K. K. Nair, A. S. Kiremidjian, Development of fragility functions as a damage classification/prediction method for steel moment-resisting frames using a wavelet-based damage sensitive feature, Earthquake Engineering \& Structural Dynamics 41 (2012) 681-696.

[24] C. Mai, K. Konakli, B. Sudret, Seismic fragility curves for structures using non-parametric representations, Frontiers of Structural and Civil Engineering 11 (2017) 169-186.

[25] I. Zentner, A general framework for the estimation of analytical fragility functions based on multivariate probability distributions, Structural Safety 64 (2017) 54-61.

[26] T. Mikosch, Copulas: Tales and facts, Extremes 9 (2006) 3-20.

[27] K. Porter, R. Kennedy, R. Bachman, Creating fragility functions for performance-based earthquake engineering, Earthquake Spectra 23 (2007) 471-489.

[28] F. Jalayer, R. De Risi, G. Manfredi, Bayesian cloud analysis: efficient structural fragility assessment using linear regression, Bulletin of Earthquake Engineering 13 (2015) 1183-1203.

[29] B. Huang, X. Du, Uncertainty analysis by dimension reduction integration and saddlepoint approximations, Journal of Mechanical Design 128 (2006) 26-33.

[30] A. Nataf, Determination des distribution don t les marges sont donnees, Comptes Rendus de 1 Academie des Sciences 225 (1962) 42-43.

[31] M. Shinozuka, Basic analysis of structural safety, Journal of Structural Engineering 109 (1983) 721-740.

[32] D. Böhning, Multinomial logistic regression algorithm, Annals of the Institute of Statistical Mathematics 44 (1992) 197-200.

[33] R. De Risi, K. Goda, S. Tesfamariam, Multi-dimensional damage measure for seismic reliability analysis, Structural Safety 78 (2019) 1-11. 
[34] N. M. Nasrabadi, Pattern recognition and machine learning, Journal of electronic imaging 16 (2007) 049901.

[35] R. K. McGuire, Probabilistic seismic hazard analysis and design earthquakes: closing the loop, Bulletin of the Seismological Society of America 85 (1995) 1275-1284.

[36] N. R. C. U. P. on Seismic Hazard Analysis, K. Aki, N. R. C. U. C. on Seismology, N. R. C. U. B. on Earth Sciences, N. R. C. U. C. on Physical Sciences, Mathematics, Resources, Probabilistic seismic hazard analysis, National Academies, 1988.

[37] E. Patelli, R. Ghanem, D. Higdon, H. Owhadi, Cossan: A multidisciplinary software suite for uncertainty quantification and risk management, Handbook of Uncertainty Quantificationt (2016) 1-69.

[38] E. Patelli, S. Tolo, H. George-Williams, J. Sadeghi, R. Rocchetta, M. De Angelis, M. Broggi, Opencossan 2.0: an efficient computational toolbox for risk, reliability and resilience analysis, Proceedings of the joint ICVRAM ISUMA UNCERTAINTIES Conference (2018).

[39] W. Goldsmith, Impact, Courier Corporation, 2001.

[40] P. Komodromos, P. C. Polycarpou, L. Papaloizou, M. C. Phocas, Response of seismically isolated buildings considering poundings, Earthquake Engineering \& Structural Dynamics 36 (2007) 1605-1622.

[41] D. Altieri, E. Tubaldi, E. Patelli, Probabilistic seismic assessment of pounding forces, in: 16th European Conference on Earthquake Engineering (2018).

[42] P. Bogacki, L. F. Shampine, A 3 (2) pair of runge-kutta formulas, Applied Mathematics Letters 2 (1989) 321-325.

[43] Y. Ohtori, R. Christenson, B. Spencer Jr, S. Dyke, Benchmark control problems for seismically excited nonlinear buildings, Journal of Engineering Mechanics 130 (2004) 366-385.

[44] D. Altieri, E. Tubaldi, E. Patelli, A. DallAsta, Assessment of optimal design methods of viscous dampers, Procedia engineering 199 (2017) $1152-1157$. 
[45] D. Altieri, E. Tubaldi, M. De Angelis, E. Patelli, A. DallAsta, Reliability-based optimal design of nonlinear viscous dampers for the seismic protection of structural systems, Bulletin of Earthquake Engineering 16 (2018) 963-982.

[46] F. McKenna, G. L. Fenves, M. H. Scott, et al., Open system for earthquake engineering simulation, University of California, Berkeley, CA (2000).

[47] A. DallAsta, E. Tubaldi, L. Ragni, Influence of the nonlinear behavior of viscous dampers on the seismic demand hazard of building frames, Earthquake Engineering \& Structural Dynamics 45 (2016) 149-169.

[48] S.-K. Au, E. Patelli, Rare event simulation in finite-infinite dimensional space, Reliability Engineering \& System Safety 148 (2016) 67-77.

[49] L. Eads, E. Miranda, H. Krawinkler, D. G. Lignos, An efficient method for estimating the collapse risk of structures in seismic regions, Earthquake Engineering \& Structural Dynamics 42 (2013) 25-41.

[50] M. Hariri-Ardebili, V. Saouma, Collapse fragility curves for concrete dams: comprehensive study, Journal of Structural Engineering 142 (2016) 04016075.

[51] G. Lupoi, P. Franchin, A. Lupoi, P. E. Pinto, Seismic fragility analysis of structural systems, Journal of Engineering Mechanics 132 (2006) 385-395.

[52] F. Jalayer, L. Elefante, I. Iervolino, G. Manfredi, Knowledge-based performance assessment of existing rc buildings, Journal of Earthquake Engineering 15 (2011) 362-389.

[53] S. Au, J. Beck, Subset simulation and its application to seismic risk based on dynamic analysis, Journal of Engineering Mechanics 129 (2003) 901-917.

[54] S. L. Kramer, Geotechnical earthquake engineering. in prentice-hall international series in civil engineering and engineering mechanics, Prentice-Hall, New Jersey (1996). 
[55] A. A. Taflanidis, J. L. Beck, Stochastic subset optimization for reliability optimization and sensitivity analysis in system design, Computers \& Structures 87 (2009) 318-331. 